\title{
Association between SARS-CoV-2 infection and disease severity among prostate cancer patients on androgen deprivation therapy: a systematic review and meta-analysis
}

\author{
Reza Sari Motlagh ${ }^{1,2} \cdot$ Mohammad Abufaraj $^{3,4} \cdot$ Pierre I. Karakiewicz $^{5} \cdot$ Pawel Rajwa $^{1,6} \cdot$ Keiichiro Mori $^{1,7}$. \\ Dong-Ho Mun ${ }^{1}$ · Shahrokh F. Shariat ${ }^{1,3,8,9,10,11,12}$
}

Received: 10 June 2021 / Accepted: 5 August 2021 / Published online: 3 September 2021

(C) The Author(s) 2021

\begin{abstract}
Purpose Androgen-regulated enzymes such as the angiotensin-converting enzyme 2 (ACE2) and the transmembrane serine protease 2 (TMPRSS2) are involved in the SARS-CoV-2 infection process. The expression of TMPRSS2 and its fusion gene, which are increased in the epithelium of the human prostate gland during prostate carcinogenesis, are regulated by androgens. Our goal was to assess the risk of the SARS-CoV-2 infection and the severity of the disease in PCa patients treated with androgen deprivation therapy (ADT).

Methods We conducted a systematic review and meta-analysis according to PRISMA guidelines. We queried PubMed and Web of Science databases on 1 July 2021. We used random- and/or fixed-effects meta-analytic models in the presence or absence of heterogeneity according to Cochrane's $Q$ test and $I^{2}$ statistic, respectively.

Results Six retrospective studies ( $n=50,220$ patients) were selected after considering inclusion and exclusion criteria for qualitative evidence synthesis. Four retrospective studies were included to assess the SARS-CoV-2 infection risk in PCa patients under ADT vs. no ADT and the summarized risk ratio (RR) was 0.8 ( $95 \%$ confidence intervals (CI) 0.44-1.47). Five retrospective studies were included to assess the severity of coronavirus disease 2019 (COVID-19) in PCa patients under ADT versus no ADT and the summarized RR was 1.23 (95\% CI 0.9-1.68).

Conclusion We found a non-significant association between the risk of SARS-CoV-2 infection and COVID-19 severity in PCa patients treated with ADT. However, our results suggest that during the COVID-19 pandemic PCa patients can safely undergo ADT as a cancer therapy without worsening COVID-19 risk and trajectory.
\end{abstract}

Keywords Androgen deprivation therapy $\cdot$ ADT $\cdot$ COVID-19 $\cdot$ Disease severity $\cdot$ SARS-CoV-2 $\cdot$ Infection risk $\cdot$ Prostate cancer

Shahrokh F. Shariat

shahrokh.shariat@meduniwien.ac.at

1 Department of Urology, Comprehensive Cancer Center, Vienna General Hospital, Medical University of Vienna, Währinger Gürtel 18-20, 1090 Vienna, Austria

2 Men's Health and Reproductive Health Research Center, Shahid Beheshti University of Medical Sciences, Tehran, Iran

3 Department of Special Surgery, Jordan University Hospital, The University of Jordan, Amman, Jordan

4 The National Center for Diabetes, Endocrinology and Genetics, The University of Jordan, Amman, Jordan

5 Cancer Prognostics and Health Outcomes Unit, University of Montreal Health Center, Montreal, Canada
6 Department of Urology, Medical University of Silesia, Zabrze, Poland

7 Department of Urology, The Jikei University School of Medicine, Tokyo, Japan

8 Institute for Urology and Reproductive Health, Sechenov University, Moscow, Russia

9 Department of Urology, Weill Cornell Medical College, New York, NY, USA

10 Department of Urology, University of Texas Southwestern, Dallas, TX, USA

11 Second Faculty of Medicine, Department of Urology, Charles University, Prague, Czech Republic

12 Karl Landsteiner Institute of Urology and Andrology, Vienna, Austria 


\section{Introduction}

The incidence of SARS-CoV-2 infection is equal in both sexes; however, disease severity and progression rates are approximately three times higher in the male gender [1-3]. This sex-specific discrepancy can potentially be explained by the mechanism of SARS-CoV-2 entry into human host cells. Both enzymes, the transmembrane angiotensinconverting enzyme 2 (ACE2) and the transmembrane protease, serine 2 (TMPRSS2) regulate SARS-CoV-2 invasion through cell membrane $[4,5]$. These enzymes are androgen-dependent and can be strongly upregulated by elevated levels of androgens $[4,6]$. Moreover, the expression of TMPRSS2 is found in the lung, gastrointestinal system, and heart $[4,6]$. The expression of TMPRSS2 and its fusion gene, which are increased in the epithelium of the human prostate gland [4] during prostate carcinogenesis, are regulated by androgens. Indeed, TMPRSS2-ERG (erythroblast-specific-related gene) gene fusion is one of the best-known aberrations in $\mathrm{PCa}$ [4] with overexpression detected in about $40-50 \%$ of PCa patients [7].

Androgen deprivation therapy (ADT) and the secondgeneration androgen receptor (AR) targeting therapy were developed to suppress the androgen-activated intracellular cascade that leads to tumor progression and aggressive tumor growth [6]. Several trials are currently evaluating androgen suppression in patients with SARS-CoV-2 infection with a focus on coronavirus disease 2019 (COVID-19) disease severity [8-12]. Since such associations can only be uncovered by very large datasets/cohorts, we aimed to aggregate data through systematic review and metaanalysis to assess the risk of SARS-CoV-2 infection and the severity of disease in PCa patients treated with ADT. Understanding either patients on ADT are at higher risk of SARS-CoV-2 infection and severe COVID-19 or there is a protective effect of ADT.

\section{Methods}

\section{Literature search}

A protocol for this study was registered a priori on the International Prospective Register of Systematic Reviews (CRD42021249405). We followed the preferred reporting items for systematic reviews and meta-analysis (PRISMA) guidelines. PubMed and Web of Science were used to search for specific queries on 1 July 2021. The search query lines and strategies were "(((ADT) OR ("androgen deprivation therapy"[All Fields])) AND ("SARS-CoV-2"[All Fields])) in PubMed database and
"ALL=(("androgen deprivation therapy" $O R$ "ADT") AND ("SARS-CoV-2" OR "COVID-19"))" in Web of Science database.

\section{Inclusion/exclusion criteria}

We only retrieved original studies published in English and excluded all other types of reports. Our main objective was to test the hypothesis stating that PCa patients who received ADT might have a lower risk of SARS-CoV-2 infection and experience a less severe form of the disease. The PICO framework items were: $\mathrm{P}$ (population) $\mathrm{PCa}$ patients and SARS-CoV-2 positive PCa patients; I (intervention group) ADT; C (control group) non-ADT; O, (outcomes) the SARS$\mathrm{CoV}-2$ infection and severe form of the disease. The severe disease was defined as ICU admission, intubation, and/or COVID-19 death. All current articles that assessed the risk of SARS-CoV-2 infection among PCa patients were eligible for this systematic review. We did not restrict our inclusion criteria to specific ADT, therefore studies analyzing GnRH agonist and antagonist, as well as oral antiandrogens, were eligible. Inclusion criteria for the quantitative meta-analysis involved all original research articles including cohort, case-control, and randomized control studies that assessed the overall risk of SARS-CoV-2 infection and severe disease as outcomes (ADT) with a control group that consisted of no ADT. Exclusion criteria involved studies without a control group (ADT).

\section{Data extraction}

Two reviewers screened the article titles and abstract screening and any disagreements about eligible and ineligible articles were resolved according to Delphi consensus criteria between co-authors. We used a data extraction sheet developed based on the Cochrane Consumers and the Communication Review Group's data extraction template (http://cccrg. cochrane.org/author-resources). We extracted the following data: first-author, type of article, year of publication, dates of the data collection or enrollment, study design, sample size, number of individuals in each study group, outcomes, how the outcomes were measured, follow-up duration, type of effect statistic and corresponding $p$ value. In the case of lacking data or doubts, we contacted articles' corresponding author(s) for additional details to overcome data limitations.

\section{Statistical analysis}

Forest plots were used to calculate and graphically depict risk ratio (RR) and summarized them to describe the RR of the SARS-CoV-2 infection and severe disease rates in the treatment and control groups. Primary and secondary metaanalyses were conducted among all studies that reported the 
SARS-CoV-2 infection and/or severe disease rates as an outcome. The heterogeneity across studies was evaluated using Cochrane's $Q$ test and $I^{2}$ statistics [13]. Significant heterogeneity was indicated by a $p \leq 0.05$ in Cochrane's $Q$ tests and a ratio $\geq 50 \%$ in $I^{2}$ statistics. We used fixed-effects models to calculate non-heterogeneous results. Random effect models were used in cases of heterogeneity. $p$ values lower than 0.05 were considered statistically significant. All analyses were carried out using Cochrane Collaboration Review Manager software (RevMan v.5.4; Cochrane Collaboration, Oxford, UK).

\section{Risk of bias}

Modified Newcastle-Ottawa Scale criteria were used to assess the quality of the included retrospective studies [14]. Above 6 points studies were considered as fair and good quality. Moreover, we used the Agency for Healthcare Research and Quality (AHRQ)[15]. The treatment and control groups of four studies were adjusted according to the potential confounding factors such as age, ischemic heart disease (IHD), hypertension, diabetes mellitus (DM), chronic obstructive pulmonary disease (COPD), and smoking status [16-19]. Montopoli et al. analyzed only ageadjusted data and their methodology was different from the other studies [20]. While all studies but Montopoli et al. divided the number of positive SARS-CoV-2 PCa patients (on or off ADT) into all SARS-CoV-2 tested PCa patients (on or off ADT), Montopoli et al. use prevalent cancer patients' data of the region registry[20]. The risk of bias and quality assessment of all studies included in the metaanalysis are summarized in Table 1.

\section{Results}

\section{Selection process}

After initial screening and excluding the duplicates, 16 articles were selected for further assessment. The search string is shown in Fig. 1. After applying the inclusion and exclusion criteria six studies (Patel et al. updated their study in
2021 [18, 21]) were included for systematic review; Caffo et al. was excluded from the meta-analysis due to the lack of a control group [22].

\section{Characteristics of included studies}

Table 2 illustrates the characteristics of the five included studies. Four and five studies reported the risk of SARSCoV-2 infection and severe COVID-19, respectively [16-21]. The reported outcomes including the odds ratio or hazard ratio of the SARS-CoV-2 infection and severe disease risks from the included studies are mentioned in Table 3.

\section{Meta-analysis}

\section{Role of SARS-CoV-2 infection risk according to ADT use}

We performed a meta-analysis, of the studies that compared the SARS-CoV-2 infection rate between ADT and no ADT PCa patients. The summarized RR of the four retrospective studies that assessed the SARS-CoV-2 infection risk (primary outcome) was 0.8 (95\% confidence intervals (CI) $0.44-1.47 ; p=0.48)$. The heterogeneity was high $\left(I^{2}=67 \%\right.$, $p=0.03$ ), so a random effect model was used. After excluding the study of Montopoli et al. that reported different results compared to the other studies, the heterogeneity decreased $\left(I^{2}=0 \%, p=0.67\right)$; the summarized RR of the three remaining studies remained statistically non-significant (RR 1.08, 95\% CI 0.77-1.51; $p=0.64$ ). The Forest plots of the meta-analysis are shown in Fig. 2A.

\section{SARS-CoV-2 disease severity according to ADT use}

We performed a meta-analysis of the studies that compared the disease severity between ADT and no ADT PCa patients. The summarized RR of five retrospective studies that assessed disease severity (secondary outcome) was 1.23 (95\% CI 0.9-1.68; $p=0.19$ ). The five studies included in the meta-analysis had a low heterogeneity $\left(I^{2}=0 \%, p=0.74\right)$, so a fixed effect model was used. The Forest plots of the metaanalysis are shown in Fig. 2B.
Table 1 The NewcastleOttawa Scale for all studies in quantitative synthesis

\begin{tabular}{llllll}
\hline Study & Selection & Compatibility & Outcome & Total & AHRQ standards \\
\hline Klein et al. [19] & $* * * *$ & $* *$ & $* *$ & 8 & Good \\
Montopoli et al. [20] & $* *$ & $* *$ & $* *$ & 6 & Fair \\
Koskinen et al. [16] & $* * * *$ & $* *$ & $* *$ & 8 & Good \\
Kwon et al. [17] & $* * * *$ & $* *$ & $* *$ & 8 & Good \\
Patel et al. [18] & $* * * *$ & $* *$ & $*$ & 7 & Good \\
\hline
\end{tabular}

$A H R Q$ Agency for Healthcare Research and Quality

Each asterisk $(*)$ represents an individual criterion within the subsection that was fulfilled 


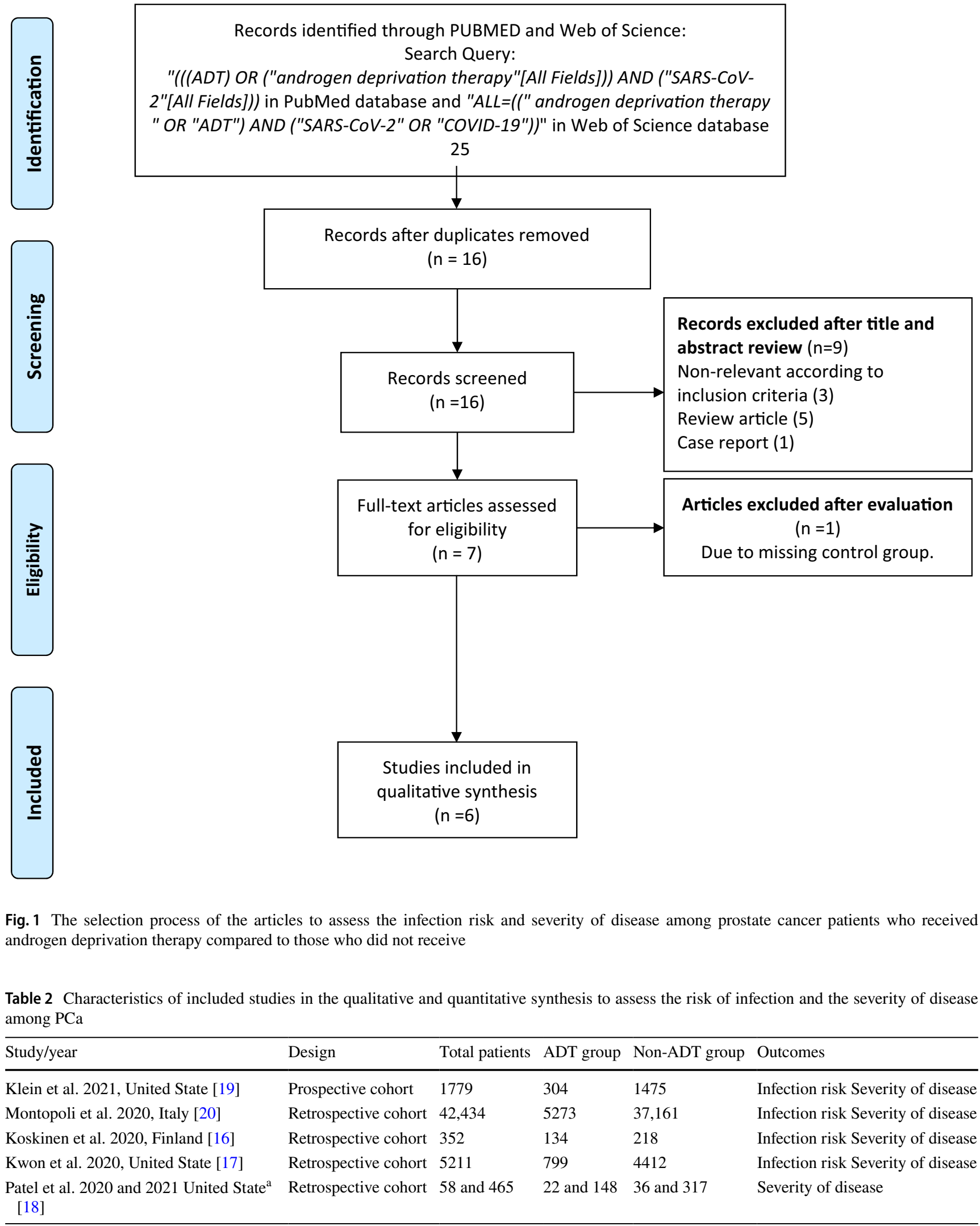

Patients who received ADT compared to who did not receive ADT

$A D T$ androgen deprivation therapy, $P C a$ prostate cancer

${ }^{\mathrm{a}}$ This study is updated in 2021 
Table 3 The reported outcomes of the included studies regarding the infection risk and severity of disease among prostate cancer patients who received ADT compared to who did not receive ADT

\begin{tabular}{|c|c|c|}
\hline Study/year/country & Infection risk for ADT & Disease severity \\
\hline Klein et al. 2021, United State [19] & OR: $0.9 ; 95 \%$ CI $0.54-1.61, p=0.8$ & Sample size limitations \\
\hline Montopoli et al. 2020, Italy [20] & OR: $4.05 ; 95 \% \mathrm{CI} ; 1.55-10.59, p=\mathbf{0 . 0 0 4 3}$ & OR: 4.40; CI 0.76-25.50, $p=0.0982$ \\
\hline Koskinen et al. 2020, Finland [16] & OR: $0.88 ; 95 \%$ CI $0.32-2.44, p=0.81$ & OR: $0.53 ; 95 \%$ CI $0.04-6.66, p=0.63$ \\
\hline Kwon et al. 2020, United State [17] & OR: $1.30 ; 95 \%$ CI $0.78-2.19, p=0.31$ & OR: $0.56,95 \%$ CI $0.07-4.88, p=0.60$ \\
\hline \multirow[t]{4}{*}{ Patel et al. 2020, United State [18] } & N/A & $\begin{array}{l}\text { Death, OR: } 0.37 ; 95 \% \text { CI } 0.08-1.80 \\
\quad p=0.220\end{array}$ \\
\hline & & $\begin{array}{l}\text { Intubation, OR: } 0.31 ; 95 \% \text { CI } 0.05-1.81 \text {, } \\
\quad p=0.192\end{array}$ \\
\hline & N/A & $\begin{array}{l}\text { Overall survival, HR } 1.28 ; 95 \% \text { CI } \\
0.79-2.08, p=0.32\end{array}$ \\
\hline & & $\begin{array}{l}\text { Intubation, HR 1.07; 95\% 0.51-2.23, } \\
\quad p=0.87\end{array}$ \\
\hline
\end{tabular}

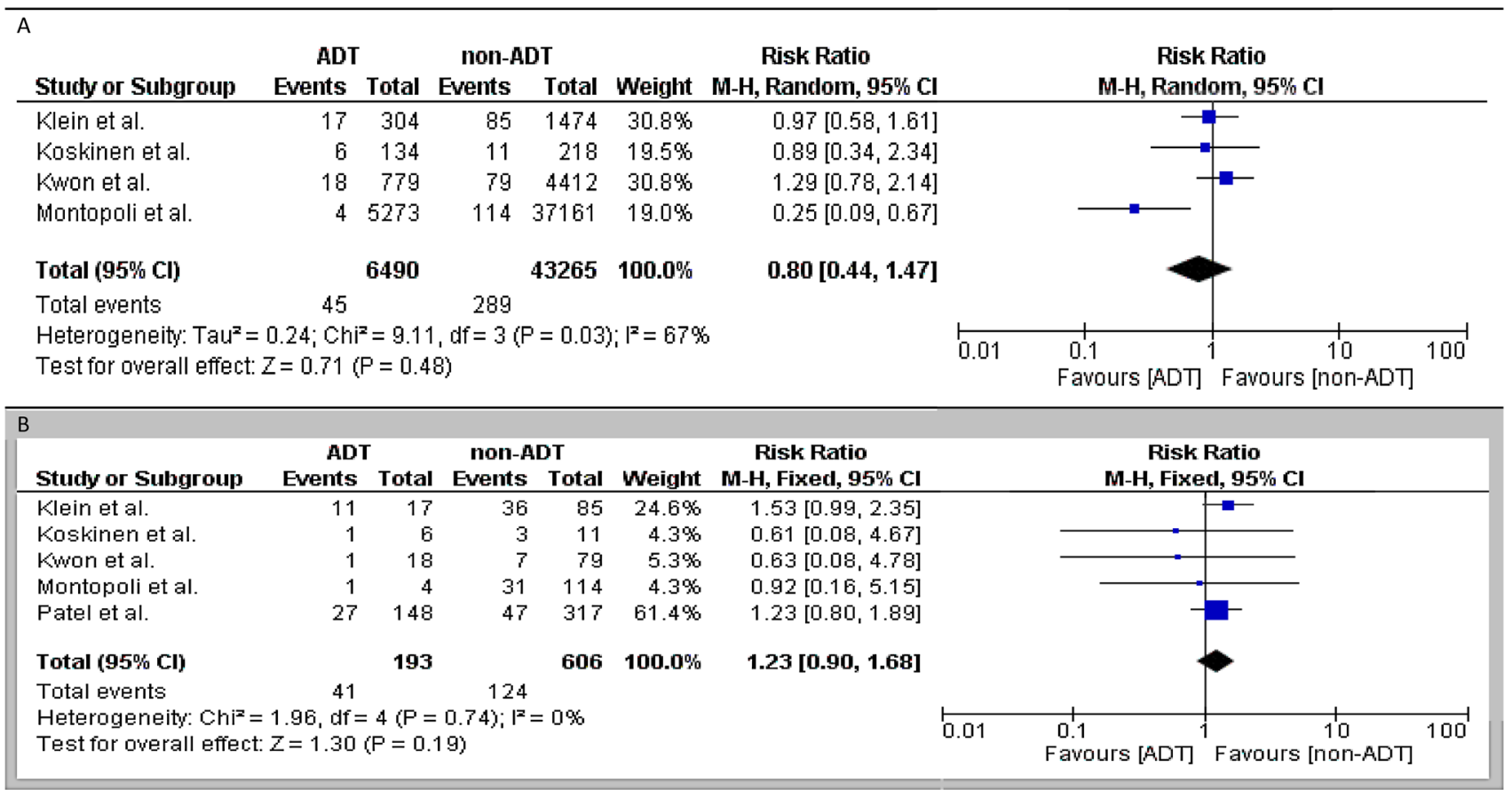

Fig. 2 Forest plot, Relative risk (RR) of the infection risk (A) and severity of disease (B) among prostate cancer patients who received ADT compared to those who did not receive ADT. ADT: androgen deprivation therapy

\section{Discussion}

The present systematic review and meta-analysis of the current cohort studies did not find a significant association between ADT use and SARS-CoV-2 infection or COVID-19 disease severity in PCa patients. The analyzed
RRs suggested an association between ADT use and the decreased risk of infection and increased severe disease; however, statistical significance was not reached. Although our results could not support the hypothesized protective effect of ADT against COVID-19 disease, there was also no increased risk of SARS-CoV-2 infection and disease 
severity among PCa patients under ADT. Therefore, the treatment of PCa patients with ADT during the COVID-19 pandemic might be safely conducted as a cancer therapy.

Our first meta-analysis result revealed high heterogeneity; thus, we tried to find the probable factors by assessment of the design of included studies. The first reports, which analyzed the SARS-CoV-2 infection risk among PCa patients under ADT suggested that androgen suppression could be protective against the SARS-CoV-2 [20]. Consequently, urological centers in the US and Finland conducted cohort studies to assess this potentially protective effect [16-19]. However, none of these could confirm a benefit to the use of ADT in reducing the risk of infection and severity of the disease. Four later cohort studies analyzed PCa patients in both ADT and no ADT groups in terms of the potential confounding factors such as IHD, COPD, hypertension, and DM; the study of Montopoli et al. did not [20]. While, Obesity, hypertension, $\mathrm{DM}$, and heart failure have been identified as risk factors of poor outcomes in both SARS-CoV-2 patients [1]. We found a low heterogeneity in the subgroup meta-analysis after excluding the Montopoli study, while the association between SARS-CoV-2 infection and ADT remained insignificant. ADT, the mainstay treatment in advanced and metastatic PCa patients, is associated with several adverse events (AEs) such as cardiac diseases and metabolic syndrome, osteoporosis, fractures, and cognitive disorders. This, indeed, can affect PCa patients' performance status and overall survival [23-25]. O'Callaghan et al. calculated that to prevent one COVID-19 case, 434 men need to be treated with ADT [26]. Thus, ADT could not provide a feasible treatment option in comparison with potential side effects.

A high rate of hospitalization was reported among metastatic castration resistance prostate cancer (mCRPC) patients who suffered from SARS-CoV-2 infection, moreover, the mortality was significantly associated with the number of previous PCa treatment lines such as different ADTs and chemotherapy [22]. Thereby, the duration of ADT and stage of disease (e.g., advanced, metastatic, CRPC, metastatic CRPC) could be the unreported confounding factor in the cohort studies that assessed the risk of SARS-CoV-2 infection and the severity of COVID-19. It has been shown that after long-term ADT and in CRPC tumors, the activity of AR remains elevated, despite reduced circulating androgen levels. Likewise, TMPRSS2 and TMPRSS2-ERG fusion genes are highly prevalent in PCa patients, including CRPC $[27,28]$. Nevertheless, the expression of TMPRSS2 and its fusion gene in the involved organs (e.g., lung, kidney, and heart) during the SARS-CoV-2 infection process have not been investigated among PCa patients treated with ADT.

The protective effect of androgen suppression has been hypothesized and case-control studies and RCTs made an effort to assess its potential effect. Up to date, the results of the RCTs are scarce to fully explore the androgen suppression effect on the treatment of the SARS-CoV-2 infection $[8-10,12]$. Additionally, the reported results of studies that assessed the protective and/or therapeutic effect of 5-alpha reductase inhibitors (5ARIs) suffer from a small sample size, the inconsistency of results, and a selection bias [29-31]. While one RCT and one case-control study found that 5ARIs reduced viral shedding/ inflammatory markers in mild to moderate COVID-19 patients and the relative risk for severe disease [29, 30]. Another population-based case-control study with a larger sample size could not show such a protective effect against COVID-19 severity[31].

The main limitation of the present systematic review and meta-analysis was the few cohort studies that assessed the risk of SARS-CoV-2 infection and COVID-19 severity among PCa patients treated with ADT. However, owing to the challenges in study design with an infectious disease it is unlikely that a good design prospective clinical trial can be performed. Moreover, up to now no study queried and mentioned this important question, which level of androgen suppression and duration are needed to show protective and/ or therapeutic effectiveness.

\section{Conclusions}

We found a non-significant association between the SARSCoV-2 infection and disease severity with ADT use among PCa patients. Although our results could not support the protective effect of ADT against SARS-CoV-2 infection and disease severity, we found that ADT does not worsen COVID-19 risk and trajectory. Indeed, ADT as a cancer treatment might be safely administered to patients during the COVID-19 pandemic. A larger sample size with adjustment of the effects of all potential confounding factors such as duration of ADT and different stages of disease (i.e. CRPC and $\mathrm{mCRPC}$ ) is necessary to further evaluate the impact of ADT on the risk and severity of SARS-CoV-2 infection. Moreover, the expression of ACE2 and TMPRSS2 in different organs and various stages of $\mathrm{PCa}$ in patients treated with ADT may help uncover the source of the biological rationale.

Acknowledgements We would like to acknowledge Dr. Vaibhav Patel from Mount Sinai University for his great attention to prepare supporting data for their study.

Author contributions RSM, MA: manuscript writing, data analysis. KM, PR: data collection. DHM: project development. PIK, SFS: manuscript editing.

Funding Open access funding provided by Medical University of Vienna. 


\section{Declarations}

\section{Conflict of interest None.}

Research involving human participants and/or animals Not applicable.

Informed consent Not applicable.

Open Access This article is licensed under a Creative Commons Attribution 4.0 International License, which permits use, sharing, adaptation, distribution and reproduction in any medium or format, as long as you give appropriate credit to the original author(s) and the source, provide a link to the Creative Commons licence, and indicate if changes were made. The images or other third party material in this article are included in the article's Creative Commons licence, unless indicated otherwise in a credit line to the material. If material is not included in the article's Creative Commons licence and your intended use is not permitted by statutory regulation or exceeds the permitted use, you will need to obtain permission directly from the copyright holder. To view a copy of this licence, visit http://creativecommons.org/licenses/by/4.0/.

\section{References}

1. O'Hearn M, Liu J, Cudhea F et al (2021) Coronavirus Disease 2019 hospitalizations attributable to cardiometabolic conditions in the united states: a comparative risk assessment analysis. J Am Heart Assoc. https://doi.org/10.1161/JAHA.120.019259

2. Peckham H, de Gruijter NM, Raine C et al (2020) Male sex identified by global COVID-19 meta-analysis as a risk factor for death and ITU admission. Nat Commun 11:6317. https://doi.org/10. 1038/s41467-020-19741-6

3. Rajwa P, Przydacz M, Krajewski W et al (2020) Changing patterns of urologic emergency visits and admissions during the COVID19 pandemic: a retrospective, multicenter, nationwide study. Arch Med Sci. https://doi.org/10.5114/aoms.2020.98364

4. Thunders M, Delahunt B (2020) Gene of the month: TMPRSS2 (transmembrane serine protease 2). J Clin Pathol 73:773-776. https://doi.org/10.1136/jclinpath-2020-206987

5. Roshandel MR, Nateqi M, Lak R et al (2020) Diagnostic and methodological evaluation of studies on the urinary shedding of SARS-CoV-2, compared to stool and serum: a systematic review and meta-analysis. Cell Mol Biol 66:148-156. https://doi.org/10. $14715 / \mathrm{cmb} / 2020.66 .6 .26$

6. Compérat E, Wasinger G, Oszwald A et al (2020) The genetic complexity of prostate cancer. Genes 11:1396. https://doi.org/10. 3390/genes11121396

7. Kumar-Sinha C, Tomlins SA, Chinnaiyan AM (2008) Recurrent gene fusions in prostate cancer. Nat Rev Cancer 8:497-511. https://doi.org/10.1038/nrc2402

8. Sidney Kimmel Comprehensive Cancer Center at Johns Hopkins (2021) A phase ii trial to promote recovery from COVID-19 with endocrine therapy. clinicaltrials.gov

9. Sidney Kimmel Comprehensive Cancer Center at Johns Hopkins (2021) COMBO trial: camostat with bicalutamide for COVID-19. clinicaltrials.gov

10. Josefsson A (2020) A prospective, multicenter, randomized phase ii clinical trial of enzalutamide treatment to decrease the morbidity in patients with corona virus disease 2019 (COVID-19). clinicaltrials.gov

11. Corpometria Institute (2021) Early antiandrogen treatment (EAT) with dutasteride for COVID-19 (EAT-DUTA AndroCoV Trial). clinicaltrials.gov
12. Applied Biology, Inc. (2021) Anti-androgen treatment for COVID-19. clinicaltrials.gov

13. Higgins JPT, Thompson SG, Deeks JJ, Altman DG (2003) Measuring inconsistency in meta-analyses. BMJ 327:557-560. https:// doi.org/10.1136/bmj.327.7414.557

14. Stang A (2010) Critical evaluation of the Newcastle-Ottawa scale for the assessment of the quality of nonrandomized studies in meta-analyses. Eur J Epidemiol 25:603-605. https://doi.org/10. 1007/s10654-010-9491-z

15. Agency for Healthcare Research and Quality (AHRQ). https:// www.ahrq.gov/. Accessed 1 Apr 2021

16. Koskinen M, Carpen O, Honkanen V et al (2020) Androgen deprivation and SARS-CoV-2 in men with prostate cancer. Ann Oncol 31:1417. https://doi.org/10.1016/j.annonc.2020.06.015

17. Kwon DH, Vashisht R, Borno HT et al (2021) Androgen deprivation therapy and SARS-CoV-2 in men with prostate cancer: findings from the University of California Health System registry. Ann Oncol. https://doi.org/10.1016/j.annonc.2021.01.067

18. Patel VG, Zhong X, Liaw B et al (2020) Does androgen deprivation therapy protect against severe complications from COVID19? Ann Oncol 31:1419-1420. https://doi.org/10.1016/j.annonc. 2020.06.023

19. Klein EA, Li J, Milinovich A et al (2021) Androgen deprivation therapy in men with prostate cancer does not affect risk of infection with SARS-CoV-2. J Urol 205:441-443. https://doi.org/10. 1097/JU.0000000000001338

20. Montopoli M, Zumerle S, Vettor R et al (2020) Androgen-deprivation therapies for prostate cancer and risk of infection by SARSCoV-2: a population-based study $(\mathrm{N}=4532)$. Ann Oncol Off J Eur Soc Med Oncol 31:1040-1045. https://doi.org/10.1016/j. annonc.2020.04.479

21. Patel VG, Zhong X, Shah NJ et al (2021) The role of androgen deprivation therapy on the clinical course of COVID-19 infection in men with prostate cancer. J Clin Oncol 39:41-41. https://doi. org/10.1200/JCO.2021.39.6_suppl.41

22. Caffo O, Gasparro D, Di Lorenzo G et al (1990) (2020) Incidence and outcomes of severe acute respiratory syndrome coronavirus 2 infection in patients with metastatic castration-resistant prostate cancer. Eur J Cancer Oxf Engl 140:140-146. https://doi.org/10. 1016/j.ejca.2020.09.018

23. Sari Motlagh R, Quhal F, Mori K et al (2021) The risk of new onset dementia and/or Alzheimer disease among patients with prostate cancer treated with androgen deprivation therapy: a systematic review and meta-analysis. J Urol 205:60-67. https://doi. org/10.1097/JU.0000000000001341

24. Abufaraj M, Iwata T, Kimura S et al (2021) Differential impact of gonadotropin-releasing hormone antagonist versus agonist on clinical safety and oncologic outcomes on patients with metastatic prostate cancer: a meta-analysis of randomized controlled trials. Eur Urol 79:44-53. https://doi.org/10.1016/j.eururo.2020.06.002

25. Smith MR, Lee WC, Brandman J et al (2005) Gonadotropinreleasing hormone agonists and fracture risk: a claims-based cohort study of men with nonmetastatic prostate cancer. J Clin Oncol Off J Am Soc Clin Oncol 23:7897-7903. https://doi.org/ 10.1200/JCO.2004.00.6908

26. O'Callaghan ME, Jay A, Kichenadasse G, Moretti KL (2020) Androgen deprivation therapy in unlikely to be effective for treatment of COVID-19. Ann Oncol 31:1780-1782. https://doi.org/10. 1016/j.annonc.2020.09.014

27. Semaan L, Mander N, Cher ML, Chinni SR (2019) TMPRSS2ERG fusions confer efficacy of enzalutamide in an in vivo bone tumor growth model. BMC Cancer 19:972. https://doi.org/10. 1186/s12885-019-6185-0

28. Blee AM, He Y, Yang Y et al (2018) TMPRSS2-ERG controls luminal epithelial lineage and antiandrogen sensitivity in PTEN and TP53-mutated prostate cancer. Clin Cancer Res Off J Am 
Assoc Cancer Res 24:4551-4565. https://doi.org/10.1158/10780432.CCR-18-0653

29. Cadegiani FA, McCoy J, Gustavo Wambier C, Goren A (2021) Early antiandrogen therapy with dutasteride reduces viral shedding, inflammatory responses, and time-to-remission in males with COVID-19: a randomized, double-blind, placebo-controlled interventional trial (EAT-DUTA AndroCoV Trial-Biochemical). Cureus. https://doi.org/10.7759/cureus.13047

30. Goren A, Wambier CG, Herrera S et al (2021) Anti-androgens may protect against severe COVID-19 outcomes: results from a prospective cohort study of 77 hospitalized men. J Eur Acad Dermatol Venereol JEADV 35:e13-e15. https://doi.org/10.1111/jdv. 16953
31. Lazzeri M, Duga S, Azzolini E et al (2021) Impact of chronic exposure to 5-alpha reductase inhibitors on the risk of hospitalization for COVID-19: a case-control study in male population from two COVID-19 regional centers of Lombardy (Italy). Minerva Urol E Nefrol Ital J Urol Nephrol. https://doi.org/10.23736/S03932249.20.04081-3

Publisher's Note Springer Nature remains neutral with regard to jurisdictional claims in published maps and institutional affiliations. 